\title{
Prevalence and Predictors of Venous Thromboembolism or Mortality in Hospitalized COVID-19 Patients
}

\author{
Stuart L. Cohen ${ }^{1,2 *}$ Eugenia Gianos ${ }^{3, *}$ Mathew A. Barish ${ }^{1}$ Saurav Chatterjee ${ }^{3,4}$ Nina Kohn ${ }^{2}$ \\ Martin Lesser ${ }^{2,4}$ Dimitrios Giannis ${ }^{2,4}$ Kevin Coppa ${ }^{5}$ Jamie S. Hirsch2,4,5 Thomas G. McGinn²,4 \\ Mark E. Goldin 2,4 Alex C. Spyropoulos ${ }^{2,4}$ for the Northwell Health COVID-19 Research Consortium
}

${ }^{1}$ Department of Radiology, Northwell Health, Manhasset, New York, United States

${ }^{2}$ Feinstein Institutes for Medical Research, Northwell Health, Manhasset, New York, United States

${ }^{3}$ Department of Cardiology, Northwell Health, Manhasset, New York, United States

${ }^{4}$ Department of Medicine, Donald and Barbara Zucker School of Medicine at Hofstra/Northwell, Northwell Health, Hempstead, New York, United States

${ }^{5}$ Department of Information Services, Northwell Health, New Hyde Park, New York, United States

Thromb Haemost 2021;121:1043-1053.
Address for correspondence Alex C. Spyropoulos, MD, FACP, FCCP, FRCPC, Department of Medicine, The Donald and Barbara Zucker School of Medicine at Hofstra/Northwell, The Institute for Health Innovations and Outcomes Research, The Feinstein Institutes for Medical Research, Anticoagulation and Clinical Thrombosis Services, Northwell Health at Lenox Hill Hospital, 130 E 77th Street, New York, NY 10075, United States (e-mail: aspyropoul@northwell.edu).

\section{Abstract}

Keywords

- COVID-19

- D-dimer

- hospitalized

- mortality

- venous thromboembolism
Background We aimed to identify the prevalence and predictors of venous thromboembolism (VTE) or mortality in hospitalized coronavirus disease 2019 (COVID-19) patients.

Methods A retrospective cohort study of hospitalized adult patients admitted to an integrated health care network in the New York metropolitan region between March 1 , 2020 and April 27, 2020. The final analysis included 9,407 patients with an overall VTE rate of $2.9 \%$ (2.4\% in the medical ward and $4.9 \%$ in the intensive care unit [ICU]) and a VTE or mortality rate of $26.1 \%$. Most patients received prophylactic-dose thromboprophylaxis. Multivariable analysis showed significantly reduced VTE or mortality with Black race, history of hypertension, angiotensin converting enzyme/angiotensin receptor blocker use, and initial prophylactic anticoagulation. It also showed significantly increased VTE or mortality with age 60 years or greater, Charlson Comorbidity Index $(\mathrm{CCl})$ of 3 or greater, patients on Medicare, history of heart failure, history of cerebrovascular disease, body mass index greater than 35 , steroid use, antirheumatologic medication use, hydroxychloroquine use, maximum D-dimer four times or greater than the upper limit of normal (ULN), ICU level of care, increasing creatinine, and decreasing platelet counts.

Conclusion In our large cohort of hospitalized COVID-19 patients, the overall inhospital VTE rate was $2.9 \%$ (4.9\% in the ICU) and a VTE or mortality rate of $26.1 \%$. Key

* Both first authors contributed equally to the production of the manuscript.

received

November 30, 2020

accepted after revision

January 18, 2021

published online

January 20, 2021
(C) 2021. Thieme. All rights reserved. Georg Thieme Verlag KG,

Rüdigerstraße 14,

70469 Stuttgart, Germany
DOI https://doi.org/ 10.1055/a-1366-9656. ISSN 0340-6245. 
predictors of VTE or mortality included advanced age, increasing $\mathrm{CCl}$, history of cardiovascular disease, ICU level of care, and elevated maximum D-dimer with a cutoff at least four times the ULN. Use of prophylactic-dose anticoagulation but not treatment-dose anticoagulation was associated with reduced VTE or mortality.

\section{Introduction}

The coronavirus disease 2019 (COVID-19) pandemic quickly led to high rates of morbidity and mortality globally, with reported rates of elevated thrombotic events, the majority of which represent venous thromboembolism (VTE). ${ }^{1}$ Inpatient rates of VTE vary (1.7-46\%), ${ }^{1-3}$ with significant mortality presumed to be secondary to VTE. ${ }^{2,3}$ These data are evidenced by postmortem studies showing classic macro-vessel disease and pulmonary microthrombi, suggesting in situ fatal pulmonary embolism $(\mathrm{PE})^{4,5}$

Predictors of VTE in COVID-19 inpatients include D-dimer, sepsis-induced coagulopathy score, lymphocyte count, and prothrombin time; these are known from generally smaller cohorts. ${ }^{6}$ A clear mechanism for the increased thrombosis rates has yet to be elucidated. Thus, optimal thromboprophylaxis strategies in high-risk COVID-19 inpatients remain unclear. While reduced VTE events and mortality have been noted with full-dose anticoagulation compared with the lowdose one, ${ }^{3,7}$ more recent studies have found either no benefit between prophylactic and therapeutic anticoagulation or that in-hospital mortality was 2.3 times greater with preemptive treatment-dose anticoagulation from the time of hospital admission. $^{8,9}$

With limited data in large populations of hospitalized COVID-19 patients to date, as well as conflicting data on predictors of VTE or mortality, we assessed the prevalence and predictors of VTE or mortality in hospitalized COVID-19 patients.

\section{Methods}

\section{Study Design, Setting, and Population}

This retrospective cohort study included adult patients with a diagnosis of COVID-19, aged 18 years and older, and hospitalized in one of 13 acute care hospitals across a multihospital integrated health care network in the New York metropolitan region between March 1,2020 and April 27,2020. Diagnosis was confirmed by a positive result on at least one polymerase chain reaction test during hospitalization. Patients were excluded if they were on the obstetrics service, or if an outcome of death or discharge had not been reached by April 30, 2020. Patients with a length of stay less than 8 hours were excluded as they did not meet the definition of inpatient stay. VTE events less than 8 hours were also excluded as those events were unlikely to be hospital-acquired. Patients were excluded if there was no baseline creatinine or platelet value, if baseline medications were not recorded, or if a Charlson Comorbidity Index (CCI) score could not be calculated. Transfers between in-system hospitals were considered as a single visit. For patients with multiple hospitalizations for COVID-19, only the first hospitalization was considered.Our system policy that went into effect on April 7, 2020 at the height of the pandemic recommended standard prophylactic-dose low-molecular-weight heparin (LMWH) for hospitalized COVID-19 patients with $\mathrm{CrCl}>15 \mathrm{~mL} / \mathrm{min}$ and intermediate doses of $\mathrm{LMWH}$ for COVID-19 inpatients with a body mass index (BMI) $>30$. The study was performed with institutional review board approval and waiver of informed consent.

\section{Data Source}

Data were obtained from the enterprise inpatient electronic health record (Sunrise Clinical Manager, Allscripts, Chicago, Illinois, United States). Data and outcomes were tracked until April 30, 2020.

\section{Outcomes}

The primary outcome of interest was a composite of first VTE event or in-hospital death. The rationale for the combined primary outcome was that death is a competing endpoint for VTE, with a large proportion of inpatient deaths attributed to undiagnosed VTE. ${ }^{5}$ Indeed, autopsy data in hospitalized patients with COVID-19 suggested that approximately 60\% and up to $100 \%$ of thrombotic events including PE and pulmonary arterial thrombosis may not be suspected before death, indicating that thrombotic mechanisms play a major role in mortality. ${ }^{4,5}$

VTE event and date were defined by new acute deep venous thrombosis (DVT) or new appearance of PE. DVTwas defined as deep vein incompressibility (where compression could be performed) or appearance of echogenic luminal material on color Doppler/duplex. ${ }^{10} \mathrm{PE}$ diagnosis was confirmed by filling defect on computed tomography pulmonary angiography. For all VTE imaging performed by the Department of Radiology, the presence or absence of VTE was prospectively entered as a discrete variable by the interpreting radiologist during the original clinical interpretation of the report beginning on April 7, 2020 or by manual consensus review of the radiology reports by two attending radiologists prior to April 7, 2020. For all point-of-care ultrasound imaging for DVT, two radiologists reviewed the extracted clinical notes documenting results. Only cases with a definitive diagnosis of DVT based on pointof-care ultrasound were recorded as positive.

\section{Covariates}

We collected data on patient demographics, comorbidities, home/hospital medications, baseline laboratory results, and intensive care unit (ICU) admission. All covariates were measured at baseline, except for in-hospital anticoagulation, 
D-dimer levels, and ICU level of care. Baseline was defined as the interval from the start of hospital care until 48 hours postadmission. Start of hospital care was defined as the earliest event of registration, admission to the hospital, or admission to the ICU.

We used patient-reported race and ethnicity to categorize patients into one of five groups: White, Black, Asian, Other/ Multiracial, and Unknown/Declined. We identified the following comorbidities by International Statistical Classification of Disease and Related Health Problems, Tenth Revision (ICD-10) coding: cancer, coronary artery disease (CAD), hypertension, asthma, chronic obstructive pulmonary disease (COPD), diabetes, chronic liver disease, peripheral arterial disease (PAD) or peripheral vascular disease (PVD), cerebrovascular disease, hyperlipidemia, end-stage renal disease (ESRD), or chronic kidney disease (CKD). We calculated the $\mathrm{CCI}$ as a measure of total comorbidity burden. Smoking history was categorized as active/former smoker, never smoker, or unknown smoker. BMI was categorized as less than or equal to 35 , greater than 35 , or unknown.

Baseline laboratory results included the first creatinine and platelet results within 48 hours of admission. Maximum D-dimer included the highest D-dimer during hospitalization for patients without VTE or highest D-dimer prior to VTE for patients with VTE. Maximum D-dimer was categorized as normal to less than 4 times the upper limit of normal (ULN), 4 to 6 times the ULN, $>6$ times the ULN, and unknown. The ULN for D-dimer was $239 \mathrm{ng} / \mathrm{mL}$.

Inpatient medications included anticoagulants, antiplatelets, steroids, intravenous immunoglobulin (IVIG), biologics, rheumatologic anti-inflammatories, immunosuppressants, antivirals, angiotensin converting enzyme (ACE)/angiotensin receptor blockers (ARBs), azithromycin, hydroxychloroquine (HCQ), chloroquine, famotidine, statins, and antacids/antihistamines. Medications started within 48 hours of admission were considered baseline medications.

Home medications included anticoagulants and antiplatelets. Thromboprophylaxis was classified as none, treatment dose, or prophylaxis dose per - Supplementary Table S1 (available in the online version). We defined in-hospital thromboprophylaxis at treatment dose only if treatment-dose anticoagulation was started more than 24 hours prior to the endpoint (VTE/death vs. discharged alive or transferred). This definition excluded patients merely with high clinical suspicion for VTE (but no objective testing). We also conducted a sensitivity analysis that included treatment-dose anticoagulation irrespective of a time cutoff. For patients diagnosed with a VTE, the highest dose prior to the first diagnosed VTE was used in all analyses. For patients not diagnosed with a VTE, the highest anticoagulant dose prior to discharge (deceased or alive) was used.

A patient was considered to have been admitted to the ICU only if there was a recorded date/time of ICU level of care (defined as use of vasopressors, ventilation, or admission to a named ICU). ICU-attributable VTE had to occur at least 2 hours after start of ICU level care; VTE within 2 hours was considered attributable to non-ICU.

\section{Data Analysis}

For each categorical factor, the chi-square test was used to examine the association between that factor and the composite outcome of VTE or death. For each continuous factor, logistic regression was used to examine the association between that factor and VTE/death.

Factors that were significantly associated with VTE/death in the univariable analysis $(p<0.10)$, or specified a priori, were included in a multivariable logistic regression model to examine the joint effects of those factors on VTE/death. Factors specified for inclusion a priori were those that literature strongly associated with VTE, including cancer, heart failure, PVD/PAD, CVD, CKD or ESRD, antiplatelet medications (either at home or started within 48 hours of admission), home anticoagulants, in-hospital anticoagulants, age, and CCI. Backward elimination was then used to remove factors that did not contribute information to the model.

All analyses were performed with SAS version 9.4 (SAS institute, Cary, North Carolina).

\section{Results}

As shown in - Fig. 1, in total 11,265 patients were considered. After 1,858 exclusions, 9,407 patients met the criteria. Of those, $63.8 \%$ were $>60$ years, $13.0 \%$ had $\mathrm{BMI} \geq 35,59.3 \%$ were males, $38.3 \%$ were white, and $21.2 \%$ were Hispanic ( - Table 1 ). CCI $\geq 5$ in $46.4 \%$ of patients, and Medicare was the most frequent insurance (47.3\%). For past medical history, $7.7 \%$ of patients had cancer, $59.9 \%$ hypertension, $12.8 \% \mathrm{CAD}, 8.2 \%$ heart failure, 4.0\% PAD/PVD, 2.6\% VTE, 5.9\% cerebrovascular disease, $20.7 \%$ hyperlipidemia, 2.5\% chronic liver disease, $8.4 \%$ asthma, $6.1 \%$ COPD, 36.1\% diabetes, and 8.3\% CKD/ESRD. Also, 7.4\% of patients were on treatment-dose home anticoagulation compared with $2.9 \%$ on prophylactic dose and $65.5 \%$ with no home anticoagulation. Further, $29.8 \%$ of patients were on hospital or home antiplatelet therapy. ICU admission was recorded for $19.7 \%$ of patients. Also, $18.6 \%$ of patients were on treatmentdose hospital thromboprophylaxis compared with $71.0 \%$ prophylactic dose, and $10.4 \%$ with no initial hospital anticoagulant thromboprophylaxis. Finally, 76 patients $(0.81 \%)$ received their first treatment dose anticoagulants within 24 hours of a VTE without initial objective testing.

\section{VTE Rate}

VTE was diagnosed in 274 (2.9\%) patients. Of these, 170 (62.0\%) had at least 1 DVT, 85 (31.0\%) had at least 1 PE, and 19 (6.9\%) had concurrent DVT and PE. ICU level of care was associated with a greater rate of VTE $(4.9 \%$; $91 / 1,854)$ than non-ICU (or unknown ICU timing) (2.4\%; 183/7,553) $(p<0.001)$. The VTE rate was $3.3 \%$ in males compared with $2.3 \%$ in females $(p=0.003)$. The VTE rate varied significantly by maximum $\mathrm{D}$-dimer $(p<0.001)$. A maximum $\mathrm{D}$-dimer $>6$ times ULN was associated with a VTE rate of $9.3 \%(226 / 2,432)$ compared with $1.9 \%(10 / 522)$ for 4 to 6 times ULN and $0.4 \%$ $(16 / 3,860)$ for 4 times the ULN.

Of the 274 patients who had at least one VTE, 28 (10.2\%) did not receive any anticoagulation prior to diagnosis, 180 


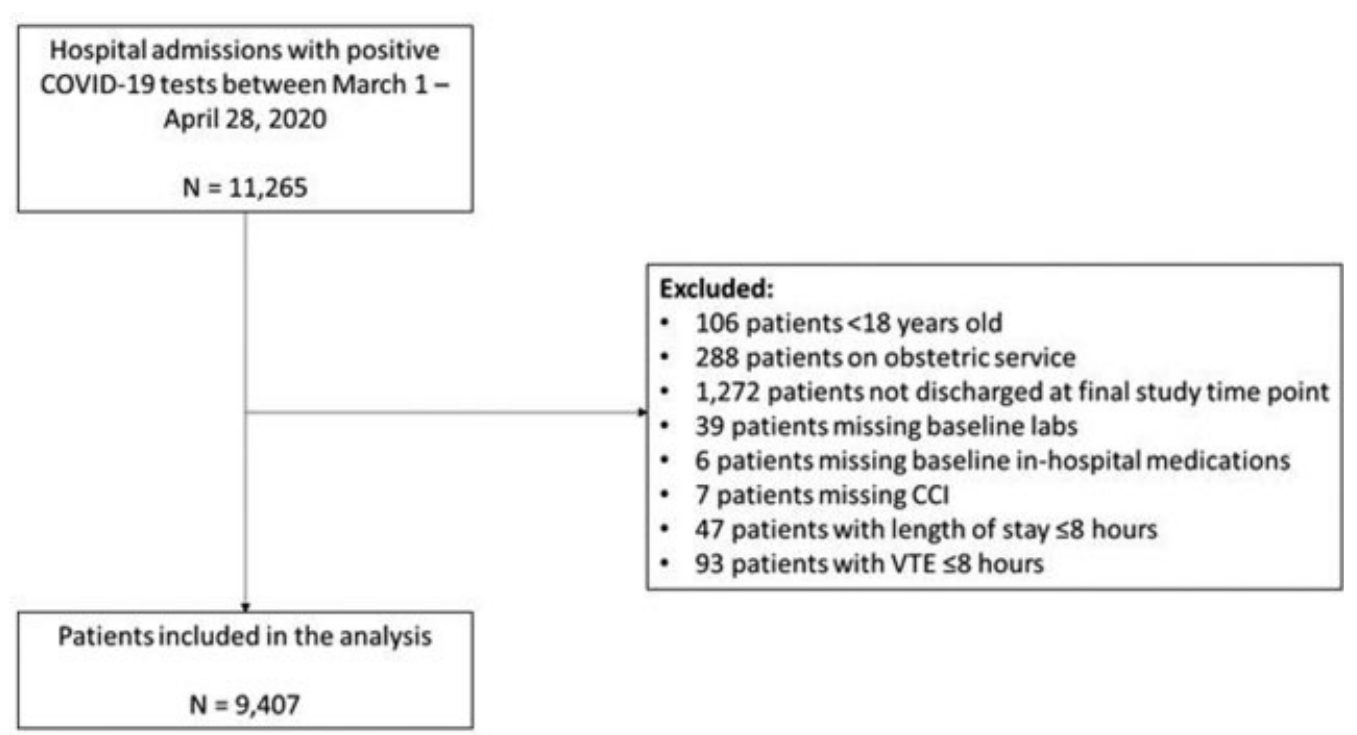

Fig. 1 Patient population. CCI, Charlson Comorbidity Index; COVID-19, coronavirus disease 2019; VTE, venous thromboembolism.

(65.7\%) received only prophylactic dose, and $66(24.1 \%)$ received treatment dose prior to diagnosis.

\section{VTE or Mortality-Univariable Analysis}

Overall VTE or mortality rate was $26.1 \%$ (-Table 2). Univariable analysis showed that the VTE or mortality rate was $27.8 \%$ in males and $23.7 \%$ in females $(p<0.001)$, and higher in ICU patients $(68.3 \%)$ than in non-ICU patients (15.3\%) $(p<0.001)$. Maximum D-dimer $>6$ times ULN was associated with a VTE or mortality rate of $55.7 \%$ compared with $29.9 \%$ for 4 to 6 times ULN and $11.4 \%$ for $<4$ times ULN (11.4\%) $(p<0.001)$. Patients with VTE or mortality had significantly higher baseline creatinine (2.1 for VTE vs. 1.5 for mortality; $p<0001)$ and significantly lower platelet counts $\left(214.5 \times 10^{9}\right.$ for VTE vs. $230.6 \times 10^{9}$ for mortality; $p<0.001$ ) than patients without VTE or mortality.

VTE or mortality was lowest with CCI $0(7.1 \%)$ compared with CCI $1-2$ (11.4\%), CCI 3-4 (21.6\%), and CCI $\geq 5$ (38.7\%) $(p<0.001)$. VTE was also lower in patients with a history of asthma $(p<0.001)$. VTE or mortality was significantly higher with history of cancer, hypertension, CAD, heart failure, PAD or PVD, VTE, cerebrovascular disease, hyperlipidemia, COPD, diabetes, and CKD/ESRD (each $p<0.001$ ).

Most in-hospital treatment regimens for COVID-19 were associated with increased rates of VTE or mortality, including steroids, IVIG, anti-inflammatories, immunosuppressants, azithromycin, and chloroquine (each $p<0.03$ ). ACE/ARB and antacids/antihistamines were associated with decreased VTE or mortality (each $p<0.001$ ). There were no significant differences in VTE or mortality with statins, famotidine, HCQ, antivirals, or biologics.

Home anticoagulation and antiplatelet use were associated with higher VTE or mortality than no anticoagulation or antiplatelet use $(p<0.001)$. The use of hospital thromboprophylaxis at prophylactic doses (but not treatment doses) was associated with a lower VTE or mortality than no anticoagulation (19.1\% for VTE vs. $30.5 \%$ for mortality; $p<0.001$ ).

\section{VTE or Mortality-Multivariable Analysis}

Multivariable analysis (- Table 3 ) showed a significant reduction in VTE or mortality with black race compared with white race (odds ratio [OR]: $0.68,95 \% \mathrm{CI}: 0.57-0.81$ ), history of hypertension (OR: 0.79, 95\% CI: 0.67-0.93), ACE/ARB use (OR: 0.75, 95\% CI: 0.62-0.92), and decreasing platelet counts (OR: 0.93 per $50,000 \times 10^{9}$ units, $95 \% \mathrm{CI}: 0.90-0.96$ ). Initial thromboprophylaxis with prophylactic-dose anticoagulation compared with no anticoagulation revealed a reduction in VTE or mortality (OR: $0.55,95 \% \mathrm{CI}$ : $0.44-0.69$ ). The use of treatment-dose anticoagulation for thromboprophylaxis did not reveal a significant reduction in VTE or mortality (OR: 0.83 , 95\% CI: 0.69-1.11). The 76 patients who received their first treatment dose of anticoagulant within 24 hours of a VTE event did not change these conclusions (data not shown).

Multivariable analysis showed a significant increase in VTE or mortality with advanced age $(60-75$ years vs. $18-59$ years [OR: $1.4,95 \%$ CI: $1.12-1.76],>75$ years vs. $18-59$ years [OR: 3.33, 95\% CI: $2.56-4.33$ ]); CCI $3-4$ versus CCI 0 (OR: $2.34,95 \% \mathrm{CI}$ : 1.54-3.55); CCI $\geq 5$ versus CCI 0 (OR: $4.99,95 \%$ CI: $3.22-7.75$ ); Medicare versus commercial insurance (OR: $1.39,95 \% \mathrm{CI}$ : 1.14-1.68); history of heart failure (OR: $1.37,95 \% \mathrm{CI}$ : 1.12-1.68); history of cerebrovascular disease (OR: $1.37,95 \%$ CI: 1.08-1.74); BMI >35 versus $\leq 35$ (OR: $1.38,95 \% \mathrm{CI}$ : 1.12-1.70); steroid use (OR: $1.68,95 \% \mathrm{CI}: 1.45-1.94)$; antirheumatic medication use (OR: 1.87, 95\% CI: 1.34-2.62); HCQ use (OR: 1.16, 95\% CI: 1.00-1.34); maximum D-dimer 4 to 6 times ULN versus 4 times the ULN (OR: $2.1,95 \% \mathrm{CI}: 1.61-2.74)$ and $>6$ times ULN versus $<4$ times ULN (OR: $5.28,95 \%$ CI: 4.46-6.25); ICU level of care (OR: 9.77, 95\% CI: 8.32-11.46); and increasing creatinine (OR: 1.03 per 0.5 units, $95 \% \mathrm{CI}$ : $1.02-1.05$ ).

\section{Discussion}

Our study of more than 9,400 COVID-19 inpatients in a multihospital health system revealed two main findings. First, the overall in-hospital symptomatic VTE rate was 
VTE in Hospitalized COVID-19 Patients Cohen et al. 1047

Table 1 Patient demographics

\begin{tabular}{|c|c|}
\hline Demographic & No. (\%) \\
\hline Total & 9,407 (100\%) \\
\hline \multicolumn{2}{|l|}{ Age, y } \\
\hline $18-59$ & $3,407(36.2 \%)$ \\
\hline $60-75$ & $3,365(35.8 \%)$ \\
\hline $75+$ & $2,635(28.0 \%)$ \\
\hline \multicolumn{2}{|l|}{ BMI } \\
\hline Unknown & $2,029(21.6 \%)$ \\
\hline$\leq 35$ & $6,154(65.4 \%)$ \\
\hline$>35$ & $1,224(13.0 \%)$ \\
\hline \multicolumn{2}{|l|}{$\mathrm{CCl}$} \\
\hline 0 & $850(9.0 \%)$ \\
\hline $1-2$ & $1,968(20.9 \%)$ \\
\hline $3-4$ & $2,222(23.6 \%)$ \\
\hline $5+$ & $4,367(46.4 \%)$ \\
\hline \multicolumn{2}{|l|}{ Sex } \\
\hline Female & $3,827(40.7 \%)$ \\
\hline Male & $5,580(59.3 \%)$ \\
\hline \multicolumn{2}{|l|}{ Race } \\
\hline Asian & $812(8.6 \%)$ \\
\hline Black & $1,995(21.2 \%)$ \\
\hline Other & $2,583(27.5 \%)$ \\
\hline Unknown & $413(4.4 \%)$ \\
\hline White & $3,604(38.3 \%)$ \\
\hline \multicolumn{2}{|l|}{ Ethnicity } \\
\hline Hispanic or Latino & $1,992(21.2 \%)$ \\
\hline Not Hispanic or Latino & $6,814(72.4 \%)$ \\
\hline Other/unknown & $601(6.4 \%)$ \\
\hline \multicolumn{2}{|l|}{ Insurance } \\
\hline Commercial & $2,810(29.9 \%)$ \\
\hline Medicaid & $1,901(20.2 \%)$ \\
\hline Medicare & 4,445 (47.3\%) \\
\hline Other & $102(1.1 \%)$ \\
\hline Self-pay & $149(1.6 \%)$ \\
\hline \multicolumn{2}{|l|}{ Medical history } \\
\hline No cancer & $8,680(92.3 \%)$ \\
\hline Cancer & 727 (7.7\%) \\
\hline No hypertension & $3,774(40.1 \%)$ \\
\hline Hypertension & $5,633(59.9 \%)$ \\
\hline No CAD & $8,207(87.2 \%)$ \\
\hline CAD & $1,200(12.8 \%)$ \\
\hline No heart failure & $8,634(91.8 \%)$ \\
\hline Heart failure & $773(8.2 \%)$ \\
\hline No PAD or PVD & $9,028(96.0 \%)$ \\
\hline
\end{tabular}

Table 1 (Continued)

\begin{tabular}{|c|c|}
\hline PAD or PVD & $379(4.0 \%)$ \\
\hline No/unknown VTE & $9,160(97.4 \%)$ \\
\hline VTE & $247(2.6 \%)$ \\
\hline No cerebrovascular disease & $8,856(94.1 \%)$ \\
\hline Cerebrovascular disease & $551(5.9 \%)$ \\
\hline No hyperlipidemia & 7,457 (79.3\%) \\
\hline Hyperlipidemia & $1,950(20.7 \%)$ \\
\hline No chronic liver disease & $9,168(97.5 \%)$ \\
\hline Chronic liver disease & $239(2.5 \%)$ \\
\hline No asthma & $8,620(91.6 \%)$ \\
\hline Asthma & $787(8.4 \%)$ \\
\hline No COPD & $8,836(93.9 \%)$ \\
\hline COPD & $571(6.1 \%)$ \\
\hline No diabetes & $6,009(63.9 \%)$ \\
\hline Diabetes & $3,398(36.1 \%)$ \\
\hline No ESRD or CKD & $8,624(91.7 \%)$ \\
\hline ESRD or CKD & $783(8.3 \%)$ \\
\hline \multicolumn{2}{|l|}{ Tobacco } \\
\hline Active/former smoker & $1,880(20.0 \%)$ \\
\hline Never smoker & $6,952(73.9 \%)$ \\
\hline Unknown smoking history & $575(6.1 \%)$ \\
\hline \multicolumn{2}{|l|}{ D-dimer maximum } \\
\hline Unknown & $2,577(27.4 \%)$ \\
\hline Normal to $<4 \times$ ULN & $3,876(41.2 \%)$ \\
\hline $4-6 \times$ ULN & $522(5.5 \%)$ \\
\hline$>6 \times$ ULN & $2,432(25.9 \%)$ \\
\hline \multicolumn{2}{|l|}{ ICU } \\
\hline No & $7,225(76.8 \%)$ \\
\hline Yes & $1,854(19.7 \%)$ \\
\hline Unknown timing & $328(3.5 \%)$ \\
\hline \multicolumn{2}{|l|}{ Treatment/medication } \\
\hline \multicolumn{2}{|l|}{ Hospital anticoagulation } \\
\hline None & 979 (10.4\%) \\
\hline Prophylaxis dose & $6,675(71.0 \%)$ \\
\hline Treatment dose & $1,753(18.6 \%)$ \\
\hline \multicolumn{2}{|l|}{ Home anticoagulation } \\
\hline Unknown & $2,274(24.2 \%)$ \\
\hline None & $6,166(65.5 \%)$ \\
\hline Prophylaxis dose & $273(2.9 \%)$ \\
\hline Treatment dose & $694(7.4 \%)$ \\
\hline \multicolumn{2}{|l|}{ Home or hospital antiplatelet } \\
\hline None & $4,531(48.2 \%)$ \\
\hline Present & $2,804(29.8 \%)$ \\
\hline NA & $2,072(22.0 \%)$ \\
\hline
\end{tabular}


Table 1 (Continued)

\begin{tabular}{|c|c|}
\hline \multicolumn{2}{|c|}{ Hospital steroids } \\
\hline None & $6,868(73.0 \%)$ \\
\hline Present & $2,539(27.0 \%)$ \\
\hline \multicolumn{2}{|c|}{ Hospital IVIG } \\
\hline None & $9,380(99.7 \%)$ \\
\hline Present & $27(0.3 \%)$ \\
\hline \multicolumn{2}{|c|}{ Hospital biologic } \\
\hline None & $9,298(98.8 \%)$ \\
\hline Present & $109(1.2 \%)$ \\
\hline \multicolumn{2}{|c|}{ Hospital rheumatologic anti-inflammatory } \\
\hline None & $9,089(96.6 \%)$ \\
\hline Present & $318(3.4 \%)$ \\
\hline \multicolumn{2}{|c|}{ Hospital immunosuppressant } \\
\hline None & $8,944(95.1 \%)$ \\
\hline Present & $463(4.9 \%)$ \\
\hline \multicolumn{2}{|c|}{ Hospital antiviral } \\
\hline None & $9,066(96.4 \%)$ \\
\hline Present & $341(3.6 \%)$ \\
\hline \multicolumn{2}{|c|}{ Hospital ACE/ARB } \\
\hline None & $8,183(87.0 \%)$ \\
\hline Present & $1,224(13.0 \%)$ \\
\hline \multicolumn{2}{|c|}{ Hospital azithromycin } \\
\hline None & $5,007(53.2 \%)$ \\
\hline Present & $4,400(46.8 \%)$ \\
\hline \multicolumn{2}{|c|}{ Hospital HCQ } \\
\hline None & $3,242(34.5 \%)$ \\
\hline Present & $6,165(65.5 \%)$ \\
\hline \multicolumn{2}{|c|}{ Hospital chloroquine } \\
\hline None & $9,391(99.8 \%)$ \\
\hline Present & $16(0.2 \%)$ \\
\hline \multicolumn{2}{|c|}{ Hospital famotidine } \\
\hline None & $8,227(87.5 \%)$ \\
\hline Present & $1,180(12.5 \%)$ \\
\hline \multicolumn{2}{|c|}{ Hospital statin } \\
\hline None & $6,283(66.8 \%)$ \\
\hline Present & $3,124(33.2 \%)$ \\
\hline \multicolumn{2}{|c|}{ Hospital antacid/antihistamine } \\
\hline None & $8,928(94.9 \%)$ \\
\hline Present & $479(5.1 \%)$ \\
\hline
\end{tabular}

Abbreviations: ACE, angiotensin converting enzyme; ARB, angiotensin receptor blockers; BMI, body mass index; $C A D$, coronary artery disease; $\mathrm{CCl}$, Charlson Comorbidity Index; COPD, chronic obstructive pulmonary disease; CKD, chronic kidney disease; ESRD, end-stage renal disease; $\mathrm{HCQ}$, hydroxychloroquine; ICU, intensive care unit; IVIG, intravenous immunoglobulin; NA, not applicable; PAD, peripheral arterial disease; PVD peripheral vascular disease; ULN, upper limit of normal, VTE, venous thromboembolism.
Table 2 Univariable predictors of VTE or mortality

\begin{tabular}{|c|c|c|c|}
\hline Predictor & $\begin{array}{l}\text { No VTE or } \\
\text { mortality, } \\
\text { No. (\%) }\end{array}$ & $\begin{array}{l}\text { VTE or } \\
\text { mortality, } \\
\text { No. (\%) }\end{array}$ & $p$-Value \\
\hline Total & $6,951(73.9 \%)$ & $2,456(26.1 \%)$ & \\
\hline \multicolumn{4}{|l|}{ Age, y } \\
\hline $18-59$ & $3,004(88.2 \%)$ & 403 (11.8\%) & \multirow[t]{3}{*}{$<0.001$} \\
\hline $60-75$ & $2,473(73.5 \%)$ & 892 (26.5\%) & \\
\hline $75+$ & $1,474(55.9 \%)$ & $1,161(44.1 \%)$ & \\
\hline \multicolumn{4}{|l|}{ BMI } \\
\hline Unknown & $1,382(68.1 \%)$ & 647 (31.9\%) & \multirow[t]{3}{*}{$<0.001$} \\
\hline$\leq 35$ & $4,624(75.1 \%)$ & $1,530(24.9 \%)$ & \\
\hline$>35$ & $945(77.2 \%)$ & $279(22.8 \%)$ & \\
\hline \multicolumn{4}{|l|}{$\mathrm{CCl}$} \\
\hline 0 & $790(92.9 \%)$ & $60(7.1 \%)$ & \multirow[t]{4}{*}{$<0.001$} \\
\hline $1-2$ & $1,744(88.6 \%)$ & $224(11.4 \%)$ & \\
\hline $3-4$ & $1,742(78.4 \%)$ & $480(21.6 \%)$ & \\
\hline $5+$ & $2,675(61.3 \%)$ & $1,692(38.7 \%)$ & \\
\hline \multicolumn{4}{|l|}{ Sex } \\
\hline Female & $2,921(76.3 \%)$ & $906(23.7 \%)$ & \multirow[t]{2}{*}{$<0.001$} \\
\hline Male & $4,030(72.2 \%)$ & $1,550(27.8 \%)$ & \\
\hline \multicolumn{4}{|l|}{ Race } \\
\hline Asian & $590(72.7 \%)$ & $222(27.3 \%)$ & \multirow[t]{5}{*}{$<0.001$} \\
\hline Black & $1,537(77.0 \%)$ & $458(23.0 \%)$ & \\
\hline Other & $2,016(78.0 \%)$ & 567 (22.0\%) & \\
\hline Unknown & $312(75.5 \%)$ & $101(24.5 \%)$ & \\
\hline White & $2,496(69.3 \%)$ & $1,108(30.7 \%)$ & \\
\hline \multicolumn{4}{|l|}{ Ethnicity } \\
\hline $\begin{array}{l}\text { Hispanic } \\
\text { or Latino }\end{array}$ & $1,565(78.6 \%)$ & 427 (21.4\%) & \multirow[t]{3}{*}{$<0.001$} \\
\hline $\begin{array}{l}\text { Not Hispanic } \\
\text { or Latino }\end{array}$ & 4,936 (72.4\%) & $1,878(27.6 \%)$ & \\
\hline Other/unknown & $450(74.9 \%)$ & $151(25.1 \%)$ & \\
\hline \multicolumn{4}{|l|}{ Insurance } \\
\hline Commercial & $2,368(84.3 \%)$ & $442(15.7 \%)$ & \multirow[t]{5}{*}{$<0.001$} \\
\hline Medicaid & $1,572(82.7 \%)$ & $329(17.3 \%)$ & \\
\hline Medicare & $2,811(63.2 \%)$ & $1,634(36.8 \%)$ & \\
\hline Other & $85(83.3 \%)$ & $17(16.7 \%)$ & \\
\hline Self-pay & 115 (77.2\%) & 34 (22.8\%) & \\
\hline \multicolumn{4}{|l|}{ Medical history } \\
\hline No cancer & $6,487(74.7 \%)$ & $2,193(25.3 \%)$ & \multirow[t]{2}{*}{$<0.001$} \\
\hline Cancer & $464(63.8 \%)$ & $263(36.2 \%)$ & \\
\hline No hypertension & $2,984(79.1 \%)$ & 790 (20.9\%) & \multirow[t]{2}{*}{$<0.001$} \\
\hline Hypertension & 3,967 (70.4\%) & $1,666(29.6 \%)$ & \\
\hline No CAD & $6,181(75.3 \%)$ & $2,026(24.7 \%)$ & \multirow[t]{2}{*}{$<0.001$} \\
\hline CAD & $770(64.2 \%)$ & $430(35.8 \%)$ & \\
\hline No heart failure & $6,524(75.6 \%)$ & $2,110(24.4 \%)$ & \multirow[t]{2}{*}{$<0.001$} \\
\hline Heart failure & 427 (55.2\%) & $346(44.8 \%)$ & \\
\hline No PAD or PVD & 6,727 (74.5\%) & $2,301(25.5 \%)$ & \multirow[t]{2}{*}{$<0.001$} \\
\hline PAD or PVD & 224 (59.1\%) & 155 (40.9\%) & \\
\hline No/unknown VTE & $6,800(74.2 \%)$ & $2,360(25.8 \%)$ & \multirow[t]{2}{*}{$<0.001$} \\
\hline VTE & 151 (61.1\%) & $96(38.9 \%)$ & \\
\hline
\end{tabular}


Table 2 (Continued)

\begin{tabular}{|c|c|c|c|}
\hline Predictor & $\begin{array}{l}\text { No VTE or } \\
\text { mortality, } \\
\text { No. (\%) }\end{array}$ & $\begin{array}{l}\text { VTE or } \\
\text { mortality, } \\
\text { No. (\%) }\end{array}$ & $p$-Value \\
\hline $\begin{array}{l}\text { No cerebrovascular } \\
\text { disease }\end{array}$ & $6,619(74.7 \%)$ & $2,237(25.3 \%)$ & \multirow[t]{2}{*}{$<0.001$} \\
\hline $\begin{array}{l}\text { Cerebrovascular } \\
\text { disease }\end{array}$ & $332(60.3 \%)$ & $219(39.7 \%)$ & \\
\hline No hyperlipidemia & $5,584(74.9 \%)$ & $1,873(25.1 \%)$ & \multirow[t]{2}{*}{$<0.001$} \\
\hline Hyperlipidemia & $1,367(70.1 \%)$ & $583(29.9 \%)$ & \\
\hline No chronic liver disease & $6,773(73.9 \%)$ & $2,395(26.1 \%)$ & \multirow[t]{2}{*}{0.835} \\
\hline Chronic liver disease & $178(74.5 \%)$ & 61 (25.5\%) & \\
\hline No asthma & $6,312(73.2 \%)$ & $2,308(26.8 \%)$ & \multirow[t]{2}{*}{$<0.001$} \\
\hline Asthma & $639(81.2 \%)$ & $148(18.8 \%)$ & \\
\hline No COPD & $6,596(74.6 \%)$ & $2,240(25.4 \%)$ & \multirow[t]{2}{*}{$<0.001$} \\
\hline COPD & $355(62.2 \%)$ & $216(37.8 \%)$ & \\
\hline No diabetes & $4,550(75.7 \%)$ & $1,459(24.3 \%)$ & \multirow[t]{2}{*}{$<0.001$} \\
\hline Diabetes & $2,401(70.7 \%)$ & $997(29.3 \%)$ & \\
\hline No ESRD or CKD & $6,433(74.6 \%)$ & $2,191(25.4 \%)$ & \multirow[t]{2}{*}{$<0.001$} \\
\hline ESRD or CKD & $518(66.2 \%)$ & $265(33.8 \%)$ & \\
\hline \multicolumn{4}{|l|}{ Tobacco } \\
\hline Active/former smoker & $1,360(72.3 \%)$ & $520(27.7 \%)$ & \multirow[t]{3}{*}{$<0.001$} \\
\hline Never smoker & $5,518(79.4 \%)$ & $1,434(20.6 \%)$ & \\
\hline $\begin{array}{l}\text { Unknown smoking } \\
\text { history }\end{array}$ & $73(12.7 \%)$ & $502(87.3 \%)$ & \\
\hline \multicolumn{4}{|l|}{ D-dimer maximum } \\
\hline Unknown & $2,075(80.5 \%)$ & $502(19.5 \%)$ & \multirow[t]{4}{*}{$<0.001$} \\
\hline Normal to $<4 \times$ ULN & $3,433(88.6 \%)$ & $443(11.4 \%)$ & \\
\hline $4-6 \times$ ULN & $366(70.1 \%)$ & $156(29.9 \%)$ & \\
\hline$>6 \times$ ULN & $1,077(44.3 \%)$ & $1,355(55.7 \%)$ & \\
\hline \multicolumn{4}{|l|}{ ICU } \\
\hline No & $6,118(84.7 \%)$ & $1,107(15.3 \%)$ & \multirow[t]{2}{*}{$<0.001$} \\
\hline Yes & $588(31.7 \%)$ & $1,266(68.3 \%)$ & \\
\hline Unknown timing & $245(74.7 \%)$ & $83(25.3 \%)$ & \\
\hline \multicolumn{4}{|l|}{ Treatment/medication } \\
\hline \multicolumn{4}{|l|}{ Hospital anticoagulation } \\
\hline None & $680(69.5 \%)$ & $299(30.5 \%)$ & \multirow[t]{3}{*}{$<0.001$} \\
\hline Prophylaxis dose & $5,398(80.9 \%)$ & $1,277(19.1 \%)$ & \\
\hline Treatment dose & $873(49.8 \%)$ & $880(50.2 \%)$ & \\
\hline \multicolumn{4}{|l|}{ Home anticoagulation } \\
\hline Unknown & $1,584(69.7 \%)$ & $690(30.3 \%)$ & \multirow[t]{4}{*}{$<0.001$} \\
\hline None & $4,741(76.9 \%)$ & $1,425(23.1 \%)$ & \\
\hline Prophylaxis dose & $175(64.1 \%)$ & $98(35.9 \%)$ & \\
\hline Treatment dose & $451(65.0 \%)$ & $243(35.0 \%)$ & \\
\hline \multicolumn{4}{|l|}{ Home or hospital antiplatelet } \\
\hline None & $3,607(79.6 \%)$ & $924(20.4 \%)$ & \multirow[t]{3}{*}{$<0.001$} \\
\hline Present & $1,857(66.2 \%)$ & $947(33.8 \%)$ & \\
\hline NA & $1,487(71.8 \%)$ & $585(28.2 \%)$ & \\
\hline \multicolumn{4}{|l|}{ Hospital steroids } \\
\hline None & $5,417(78.9 \%)$ & $1,451(21.1 \%)$ & \multirow[t]{2}{*}{$<0.001$} \\
\hline Present & $1,534(60.4 \%)$ & $1,005(39.6 \%)$ & \\
\hline \multicolumn{4}{|l|}{ Hospital IVIG } \\
\hline None & $6,936(73.9 \%)$ & $2,444(26.1 \%)$ & 0.030 \\
\hline
\end{tabular}

Table 2 (Continued)

\begin{tabular}{|c|c|c|c|}
\hline Predictor & $\begin{array}{l}\text { No VTE or } \\
\text { mortality, } \\
\text { No. (\%) }\end{array}$ & $\begin{array}{l}\text { VTE or } \\
\text { mortality, } \\
\text { No. (\%) }\end{array}$ & $p$-Value \\
\hline Present & $15(55.6 \%)$ & $12(44.4 \%)$ & \\
\hline \multicolumn{4}{|c|}{ Hospital biologic } \\
\hline None & 6,872 (73.9\%) & $2,426(26.1 \%)$ & \multirow[t]{2}{*}{0.735} \\
\hline Present & 79 (72.5\%) & $30(27.5 \%)$ & \\
\hline \multicolumn{4}{|c|}{ Hospital rheumatologic anti-inflammatory } \\
\hline None & $6,795(74.8 \%)$ & $2,294(25.2 \%)$ & \multirow[t]{2}{*}{$<0.001$} \\
\hline Present & $156(49.1 \%)$ & 162 (50.9\%) & \\
\hline \multicolumn{4}{|c|}{ Hospital immunosuppressant } \\
\hline None & $6,664(74.5 \%)$ & $2,280(25.5 \%)$ & \multirow[t]{2}{*}{$<0.001$} \\
\hline Present & $287(62.0 \%)$ & $176(38.0 \%)$ & \\
\hline \multicolumn{4}{|c|}{ Hospital antiviral } \\
\hline None & $6,700(73.9 \%)$ & $2,366(26.1 \%)$ & \multirow[t]{2}{*}{0.903} \\
\hline Present & $251(73.6 \%)$ & $90(26.4 \%)$ & \\
\hline \multicolumn{4}{|c|}{ Hospital ACE/ARB } \\
\hline None & $5,974(73.0 \%)$ & $2,209(27.0 \%)$ & \multirow[t]{2}{*}{$<0.001$} \\
\hline Present & $977(79.8 \%)$ & 247 (20.2\%) & \\
\hline \multicolumn{4}{|c|}{ Hospital azithromycin } \\
\hline None & $3,787(75.6 \%)$ & $1,220(24.4 \%)$ & \multirow[t]{2}{*}{$<0.001$} \\
\hline Present & $3,164(71.9 \%)$ & $1,236(28.1 \%)$ & \\
\hline \multicolumn{4}{|c|}{ Hospital HCQ } \\
\hline None & $2,429(74.9 \%)$ & $813(25.1 \%)$ & \multirow[t]{2}{*}{0.099} \\
\hline Present & $4,522(73.3 \%)$ & $1,643(26.7 \%)$ & \\
\hline \multicolumn{4}{|c|}{ Hospital chloroquine } \\
\hline None & $6,946(74.0 \%)$ & $2,445(26.0 \%)$ & \multirow[t]{2}{*}{$<0.001$} \\
\hline Present & $5(31.3 \%)$ & $11(68.8 \%)$ & \\
\hline \multicolumn{4}{|c|}{ Hospital famotidine } \\
\hline None & $6,072(73.8 \%)$ & $2,155(26.2 \%)$ & \multirow[t]{2}{*}{0.616} \\
\hline Present & $879(74.5 \%)$ & $301(25.5 \%)$ & \\
\hline \multicolumn{4}{|c|}{ Hospital statin } \\
\hline None & $4,653(74.1 \%)$ & $1,630(25.9 \%)$ & \multirow[t]{2}{*}{0.605} \\
\hline Present & $2,298(73.6 \%)$ & $826(26.4 \%)$ & \\
\hline \multicolumn{4}{|c|}{ Hospital antacid/antihistamine } \\
\hline None & $6,551(73.4 \%)$ & $2,377(26.6 \%)$ & \multirow[t]{2}{*}{$<0.001$} \\
\hline Present & $400(83.5 \%)$ & $79(16.5 \%)$ & \\
\hline
\end{tabular}

Abbreviations: ACE, angiotensin converting enzyme; $A R B$, angiotensin receptor blockers; BMI, body mass index; $C A D$, coronary artery disease; $\mathrm{CCl}$, Charlson Comorbidity Index; COPD, chronic obstructive pulmonary disease; CKD, chronic kidney disease; ESRD, end stage renal disease; $\mathrm{HCQ}$, hydroxychloroquine; ICU, intensive care unit; IVIG, intravenous immunoglobulin; NA, not applicable; PAD, peripheral arterial disease; PVD peripheral vascular disease; ULN, upper limit of normal, VTE, venous thromboembolism.

$2.9 \%$ and significantly higher in the ICU versus medical ward (4.9 vs. $2.4 \% ; p<0.001$ ). Second, the overall VTE or mortality rate of $26.1 \%$ was significantly associated with key clinical and laboratory predictors, including advanced age, increasing CCI, ICU care, BMI greater than 35, a history of heart failure or cerebrovascular disease, and maximum D-dimer $>4$ times (and especially $>6$ times) ULN for our laboratory. Prophylactic-dose anticoagulation, but not treatment-dose 
Table 3 Multivariable predictors of VTE or mortality

\begin{tabular}{|c|c|c|c|}
\hline & OR & $95 \% \mathrm{Cl}$ & $p$-Value \\
\hline \multicolumn{4}{|l|}{ Age, y } \\
\hline $18-59$ & & Reference & \\
\hline $60-75$ & 1.40 & $(1.12,1.76)$ & 0.003 \\
\hline$>75$ & 3.33 & $(2.56,4.33)$ & $<0.001$ \\
\hline \multicolumn{4}{|l|}{ BMI } \\
\hline$\leq 35$ & & Reference & \\
\hline$>35$ & 1.38 & $(1.12,1.70)$ & 0.003 \\
\hline Unknown & 1.33 & $(1.14,1.55)$ & $<0.001$ \\
\hline \multicolumn{4}{|l|}{$\mathrm{CCl}$} \\
\hline 0 & & Reference & \\
\hline $1-2$ & 1.46 & $(0.99,2.13)$ & 0.054 \\
\hline $3-4$ & 2.34 & $(1.54,3.55)$ & $<0.001$ \\
\hline $5+$ & 4.99 & $(3.22,7.75)$ & $<0.001$ \\
\hline \multicolumn{4}{|l|}{ Sex } \\
\hline Female & & Reference & \\
\hline Male & 1.21 & $(1.06,1.38)$ & 0.006 \\
\hline \multicolumn{4}{|l|}{ Race } \\
\hline White & & Reference & \\
\hline Asian & 1.08 & $(0.84,1.38)$ & 0.545 \\
\hline Black & 0.68 & $(0.57,0.81)$ & $<0.001$ \\
\hline Other & 0.81 & $(0.68,0.96)$ & 0.014 \\
\hline Unknown & 0.87 & $(0.63,1.22)$ & 0.425 \\
\hline \multicolumn{4}{|l|}{ Insurance } \\
\hline Commercial & & Reference & \\
\hline Medicaid & 1.16 & $(0.93,1.44)$ & 0.186 \\
\hline Medicare & 1.39 & $(1.14,1.68)$ & 0.001 \\
\hline Other & 1.33 & $(0.68,2.62)$ & 0.404 \\
\hline Self-pay & 1.14 & $(0.63,2.06)$ & 0.668 \\
\hline \multicolumn{4}{|l|}{ Medical history } \\
\hline Hypertension & 0.79 & $(0.67,0.93)$ & 0.005 \\
\hline Heart failure & 1.37 & $(1.12,1.68)$ & 0.003 \\
\hline Cerebrovascular disease & 1.37 & $(1.08,1.74)$ & 0.009 \\
\hline \multicolumn{4}{|l|}{ Tobacco } \\
\hline Never & & Reference & \\
\hline Active/former smoker & 1.00 & $(0.86,1.17)$ & 1.000 \\
\hline Unknown & 15.48 & $(11.45,20.92)$ & $<0.001$ \\
\hline \multicolumn{4}{|l|}{ D-dimer maximum } \\
\hline Normal- $4 \times$ ULN & & Reference & \\
\hline $4-6 x$ ULN & 2.1 & $(1.61,2.74)$ & $<0.001$ \\
\hline$>6 x$ ULN & 5.28 & $(4.46,6.25)$ & $<0.001$ \\
\hline Unknown & 1.96 & $(1.63,2.34)$ & $<0.001$ \\
\hline \multicolumn{4}{|l|}{ ICU } \\
\hline No & & Reference & \\
\hline Unknown & 1.42 & $(1.02,1.98)$ & 0.036 \\
\hline Yes & 9.77 & $(8.32,11.46)$ & $<0.001$ \\
\hline
\end{tabular}

Table 3 (Continued)

\begin{tabular}{|c|c|c|c|}
\hline \multicolumn{4}{|l|}{ Treatment/medication } \\
\hline Steroid use & 1.68 & $(1.45,1.94)$ & $<0.001$ \\
\hline $\begin{array}{l}\text { Rheumatologic } \\
\text { anti-inflammatory }\end{array}$ & 1.87 & $(1.34,2.62)$ & $<0.001$ \\
\hline $\mathrm{ACE} / \mathrm{ARB}$ & 0.75 & $(0.62,0.92)$ & 0.006 \\
\hline HCQ & 1.16 & $(1.00,1.34)$ & 0.047 \\
\hline \multicolumn{4}{|l|}{ Home anticoagulation } \\
\hline None & & Reference & \\
\hline Prophylactic dose & 1.20 & $(0.86,1.67)$ & 0.279 \\
\hline Treatment dose & 0.88 & $(0.69,1.11)$ & 0.273 \\
\hline Unknown & 1.69 & $(1.42,2.01)$ & $<0.001$ \\
\hline \multicolumn{4}{|l|}{ Hospital anticoagulation } \\
\hline None & & Reference & \\
\hline Prophylactic dose & 0.55 & $(0.44,0.69)$ & $<0.001$ \\
\hline Treatment dose & 0.83 & $(0.65,1.07)$ & 0.152 \\
\hline \multicolumn{4}{|l|}{ Baseline laboratory } \\
\hline $\begin{array}{l}\text { Creatinine (per } 0.5 \text { unit } \\
\text { increase) }\end{array}$ & 1.03 & $(1.02,1.05)$ & $<0.001$ \\
\hline $\begin{array}{l}\text { Platelet count (per } \\
50,000 \text { unit increase) }\end{array}$ & 0.93 & $(0.90,0.96)$ & $<0.001$ \\
\hline
\end{tabular}

Abbreviations: $\mathrm{Cl}$, confidence interval; OR, odds ratio.

Note: See - Table 1.

anticoagulation, was significantly associated with a decrease in VTE or mortality.

The overall in-hospital symptomatic VTE rate of $2.9 \%$ ( $4.9 \%$ in ICU) in our COVID-19 population was slightly higher than VTE rates usually found in medical and ICU wards for patients with sepsis and pneumonia. However, it is also an order of magnitude less than the reported incidence of VTE of 27 to $46 \%$ in China and Western Europe without systematic screening. ${ }^{1,3,11,12}$ Indeed, the VTE rate of $2.4 \%$ in the non-ICU population of our study was more in line with the rates of 3.6 and $1.7 \%$ reported in hospitalized COVID-19 populations in New York City health systems during the early part of the pandemic., ${ }^{2,6}$ The reasons for the lower VTE rates in U.S. health systems are unclear. Our cohort is much larger than those described previously; we restricted our analysis to capture only clinically evident in-hospital events; we excluded other forms of thrombosis, such as catheter thrombosis, which were included in some studies, and, importantly, our system established early a universal thromboprophylaxis policy for COVID-19 inpatients. ${ }^{13}$

Our analysis revealed a high in-hospital VTE or mortality rate of $26.1 \%$, which is similar to rates of thrombosis and mortality of 30 to $42 \%$ in previous studies. ${ }^{6,14}$ For our analysis of in-hospital predictors, we combined VTE and mortality because autopsy data in hospitalized patients with COVID-19 suggested that up to approximately $60 \%$ of VTE was not suspected before death. These data indicated that thrombotic mechanisms play a major role in mortality and that all-cause mortality and VTE represent competing risks. ${ }^{5,15}$ Significant key clinical predictors of in-hospital VTE or mortality included advanced age, especially $>75$ years (OR: 3.3 ), a CCI $\geq 5$ (OR: 
4.99), history of heart failure or cerebrovascular disease (both with OR: 1.37), BMI $\geq 35$ (OR: 1.38), and ICU level of care (OR: 9.77). Advanced age, history of cardiovascular disease, and obesity have been consistently associated with poor outcomes and increased thrombotic events and mortality in hospitalized COVID-19 patients. ${ }^{16}$ Our study is the first to show a nearly fivefold increased risk of VTE or mortality with an elevated CCI $\geq 5$ in this population. Our study showed a nearly 10 -fold increased risk of VTE or mortality in critically ill COVID-19 patients. ICU level of care has consistently been a predictor of elevated risk of thromboembolic disease and mortality in this population. ${ }^{6,17}$ Other clinical predictors of VTE or mortality included steroid use (OR: 1.68), antirheumatic medication (OR: 1.87), and HCQ use (OR: 1.16), as well as Medicare coverage (OR: 1.39). Black race appeared protective for VTE or mortality, consistent with a previous report that poverty but not race is associated with severe disease. ${ }^{18}$ Causal mechanisms for these associations warrant further study.

Elevated D-dimer, either four to six times ULN (OR: 2.1) or $\geq 6$ times ULN (OR: 5.28 ), showed a significant correlation with VTE or mortality. Early reports from China suggested elevated D-dimer cutoffs of four and, especially, six times ULN based on local laboratory criteria were strong predictors of mortality in COVID-19 inpatients. This observation has been corroborated in our study and by other groups. ${ }^{2,3}$ Highly elevated D-dimer may reflect the hyperinflammatory state and cytokine storm that lead to thromboinflammation..$^{19}$ We tested and did not find significant associations between VTE or mortality with other laboratory criteria and coagulation parameters that were previously (but not consistently) shown to be predictors of poor outcomes. ${ }^{3}$ Increasing creatinine and decreasing platelet counts were associated with minimal clinical implications for VTE or mortality.

In our study, the use of initial prophylactic-dose anticoagulation compared with no anticoagulation was significantly associated with a $45 \%$ reduction in VTE or mortality. This finding is consistent with an older report from China that suggested a $39 \%$ reduction with prophylactic-dose anticoagulant therapy versus no anticoagulant therapy COVID-19 inpatients with elevated D-dimer. ${ }^{3}$ It is also consistent with a recent New York area study that revealed an in-hospital mortality of $29.1 \%$ for ICU patients treated with anticoagulants versus $62.7 \%$ in patients without anticoagulants. ${ }^{3,7}$ Interestingly, we did not find a significant reduction in VTE or mortality with initial treatment-dose anticoagulation. Some reports suggest advantages of using treatment-dose anticoagulant therapy over prophylactic dose for thromboprophylaxis. ${ }^{20}$ More recent reports suggest no significant advantages in reducing thrombotic events or mortality. ${ }^{7}$ Ongoing randomized trials are comparing usual prophylactic-dose heparin therapy to escalated or treatment-dose heparin therapy for optimal thromboprophylaxis in hospitalized and critically ill COVID-19 patients, with all-cause mortality as the key endpoint. ${ }^{21}$

Our study has several limitations. Although VTE events were carefully adjudicated by two radiologists, the true VTE rate may be underreported as VTE events may not have had confirmatory imaging studies due to concerns of virus exposure by health care staff. In addition, our institutional policy did not advocate for systematic DVT screening, especially in critically ill patients, which may have underrepresented the true VTE incidence. ${ }^{11}$ We also were not able to differentiate in situ versus embolic PE with our dataset. We did not include arterial thromboembolism or device- or catheter-associated thrombosis, which may have underrepresented the true incidence of macro-vessel thrombotic disease. In the absence of autopsy data or from objective exam testing, we recognize that mechanisms other than thrombotic ones may have contributed to mortality. However, the overall lower rates of VTE in our data and other recent regional data may point to either an ascertainment bias seen in previous reports of the incidence of COVID-19-induced coagulopathy or in different clinical manifestations of different COVID-19 genotypes. Data collection with critically ill patients may have been fragmented due to limited history and may explain why key clinical data were unavailable in patients with worse outcomes. We could not rule out hidden confounders when attempting to develop our multivariable model. A last potential limitation is that the primary outcome of the composite of VTE or death was analyzed as a binary outcome, using logistic regression rather than survival analysis, even though the endpoint is actually a "time-until-event" outcome. However, from a clinical and practical perspective, the goal of treatment for hospitalized COVID-19 patients is to improve the patient's condition and discharge that patient alive. The length of time it takes to achieve that outcome is not of paramount interest, and thus the use of the binary outcome is reasonable.

Despite these limitations, our analysis has several strengths. It represents a very large in-hospital dataset of hospitalized COVID-19 patients, with over 9,400 patients, allowing more precise estimates of VTE and mortality than previous studies. The VTE events were carefully and systematically adjudicated by experienced radiologists, allowing for greater specificity. We used a centralized COVID-19 database, which was available soon after the pandemic struck our system, that allowed for uniformity of data definitions of both clinical and laboratory factors. The high rate of our combined outcome of VTE and mortality allowed us to adequately assess significant associations with independent variables in the univariable and multivariable analyses.

\section{Conclusion}

Our study of over 9,400 hospitalized COVID-19 patients found an overall in-hospital VTE rate of $2.9 \%$ (4.9\% in ICU) and an overall VTE or mortality rate of $26.1 \%$. Key predictors of VTE or mortality included advanced age, increasing CCI, history of cardiovascular disease, ICU level of care, and elevated maximum D-dimer with a cutoff of at least four times ULN. Use of prophylactic-dose anticoagulation (vs. no anticoagulation) but not treatment-dose anticoagulation was associated with a reduction of VTE or mortality. Our results support universal in-hospital thromboprophylaxis using standard prophylacticdose anticoagulants for hospitalized COVID-19 patients, with potential to use individual clinical and laboratory parameters to develop a predictive score for VTE and mortality to tailor individualized thromboprophylaxis strategies in high-risk subgroups. 


\section{One-Sentence Summary}

Key predictors of venous thromboembolism (VTE) or mortality in a large hospitalized coronavirus disease 2019 (COVID-19) population support universal prophylactic-dose thromboprophylaxis with potential to use individual clinical and laboratory parameters to develop a predictive score for individualized thromboprophylaxis strategies in high-risk subgroups.

\section{What is known about this topic?}

- Hospitalized COVID-19 patients have considerable rates of venous thromboembolism and thromboembolism-associated mortality.

- Optimal thromboprophylaxis strategies in high-risk COVID-19 inpatients remain unclear.

- Reduced VTE events and mortality have been noted with full-dose anticoagulation compared with the lowdose one.

\section{What does this paper add?}

- Identification of key predictors of venous thromboembolism or mortality in a large hospitalized COVID-19 population.

- Universal prophylactic-dose thromboprophylaxis should be considered in hospitalized patients with COVID-19.

- Individual clinical and laboratory parameters may be used to develop a predictive score for individualized thromboprophylaxis strategies in high-risk subgroups.

Note

The data that support the findings of this study are available on request from http://COVID19@northwell. edu. The data are not publicly available due to restrictions as it could compromise the privacy of research participants. This was performed at Northwell Health.

Funding

This work was supported by the Association of University Radiologists GE Radiology Research Academic Fellowship (GERRAF) and Siemens Healthineers. S.L.C. received an honorarium for an educational presentation for Siemens Healthineers in 2020 and was a consultant for Infervision 2019. This work was also supported by the National Institute on Aging of the National Institutes of Health (R24AG064191) and the National Library of Medicine of the National Institutes of Health (R01LM012836).

\section{Conflict of Interest}

A.C.S.: consultant for Janssen, Bayer, Bristol Meyers Squibb, Boehringer Ingelheim, the ATLAS Group, and research grants from Janssen and Boehringer Ingelheim. S.L.C.: consultant for Infervision, educational honorarium from Siemens Healthineers, and research funding from the Association of University Radiologists GE Radiology Research Academic Fellowship (GERRAF) and Siemens Healthineers. None of the other authors reported any conflicts of interest.

\section{Acknowledgments}

We would like to acknowledge Karina W. Davidson and the Northwell Health COVID-19 Research Consortium and the Feinstein Institutes for Medical Research, as well as all the frontline providers and patients who made this work possible during the height of the COVID-19 pandemic in New York City.

\section{References}

1 Klok FA, Kruip MJHA, van der Meer NJM, et al. Confirmation of the high cumulative incidence of thrombotic complications in critically ill ICU patients with COVID-19: An updated analysis. Thromb Res 2020;191:148-150

2 Hanif A, Khan S, Mantri N, et al. Thrombotic complications and anticoagulation in COVID-19 pneumonia: a New York City hospital experience. Ann Hematol 2020;99(10):2323-2328

3 Tang N, Bai H, Chen X, Gong J, Li D, Sun Z. Anticoagulant treatment is associated with decreased mortality in severe coronavirus disease 2019 patients with coagulopathy. J Thromb Haemost 2020;18(05):1094-1099

4 Lax SF, Skok K, Zechner P, et al. Pulmonary arterial thrombosis in COVID-19 with fatal outcome: results from a prospective, singlecenter, clinicopathologic case series. Ann Intern Med 2020;173 (05):350-361

5 Wichmann D, Sperhake J-P, Lütgehetmann M, et al. Autopsy findings and venous thromboembolism in patients with COVID19: a prospective cohort study. Ann Intern Med 2020;173(04): 268-277

6 Bilaloglu S, Aphinyanaphongs Y, Jones S, Iturrate E, Hochman J, Berger JS. Thrombosis in hospitalized patients with COVID-19 in a New York City health system. JAMA 2020;324(08):799-801

7 Paranjpe I, Fuster V, Lala A, et al. Association of treatment dose anticoagulation with in-hospital survival among hospitalized patients with COVID-19. J Am Coll Cardiol 2020;76(01):122-124

8 Motta JK, Ogunnaike RO, Shah R, et al. Clinical outcomes with the use of prophylactic versus therapeutic anticoagulation in COVID-19. Crit Care Explor 2020;2(12):e0309

9 Nadkarni GN, Lala A, Bagiella E, et al. Anticoagulation, bleeding, mortality, and pathology in hospitalized patients with COVID-19. J Am Coll Cardiol 2020;76(16):1815-1826

10 Needleman L, Cronan JJ, Lilly MP, et al. Ultrasound for lower extremity deep venous thrombosis: Multidisciplinary recommendations from the Society of Radiologists in Ultrasound Consensus Conference. Circulation 2018;137(14):1505-1515

11 Cui S, Chen S, Li X, Liu S, Wang F. Prevalence of venous thromboembolism in patients with severe novel coronavirus pneumonia.J Thromb Haemost 2020;18(06):1421-1424

12 Lodigiani C, Iapichino G, Carenzo L, et al; Humanitas COVID-19 Task Force. Venous and arterial thromboembolic complications in COVID-19 patients admitted to an academic hospital in Milan, Italy. Thromb Res 2020;191:9-14

13 Cohoon KP, Mahé G, Tafur AJ, Spyropoulos AC. Emergence of institutional antithrombotic protocols for coronavirus 2019. Res Pract Thromb Haemost 2020;4(04):510-517

14 Bhatraju PK, Ghassemieh BJ, Nichols M, et al. Covid-19 in critically ill patients in the Seattle region - case series. N Engl J Med 2020; 382(21):2012-2022

15 Fox SE, Akmatbekov A, Harbert JL, Li G, Quincy Brown J, Vander Heide RS. Pulmonary and cardiac pathology in African American 
patients with COVID-19: an autopsy series from New Orleans. Lancet Respir Med 2020;8(07):681-686

16 Katz MH. Regardless of age, obesity and hypertension increase risks with COVID-19. JAMA Intern Med 2020. Doi: 10.1001/ jamainternmed.2020.5415

17 Poissy J, Goutay J, Caplan M, et al; Lille ICU Haemostasis COVID-19 Group. Pulmonary embolism in patients with COVID-19: awareness of an increased prevalence. Circulation 2020;142(02): 184-186

18 Muñoz-Price LS, Nattinger AB, Rivera F, et al. Racial disparities in incidence and outcomes among patients with COVID-19. JAMA Netw Open 2020;3(09):e2021892
19 Spyropoulos AC, Weitz JI. Hospitalized COVID-19 patients and venous thromboembolism: a perfect storm. Circulation 2020;142 (02):129-132

20 Llitjos J-F, Leclerc M, Chochois C, et al. High incidence of venous thromboembolic events in anticoagulated severe COVID-19 patients. J Thromb Haemost 2020;18(07):1743-1746

21 Tritschler T, Mathieu M-E, Skeith L, et al; International Network of VENous Thromboembolism Clinical Research Networks INVENTVTE. Anticoagulant interventions in hospitalized patients with COVID-19: a scoping review of randomized controlled trials and call for international collaboration. J Thromb Haemost 2020;18 (11):2958-2967 